\title{
Metabolism and metabolic inhibition of gambogic acid in rat liver microsomes $^{1}$
}

\author{
Yi-tong LIU, Kun HAO, Xiao-quan LIU², Guang-ji WANG \\ Key Lab of Drug Metabolism and Pharmacokinetics, China Pharmaceutical University, Nanjing 210009, China
}

\section{Key words}

gambogic acid; metabolism; cytochrome $\mathrm{P}$ 450; liver microsomes; metabolic inhibition

${ }^{1}$ Project supported by the National High Technology "863" Project (№ 2003AA2Z347A), and Jiangsu Key Lab of Drug Metabolism and Pharmacokinetics (No BM2001201).

${ }^{2}$ Correspondence to Prof Xiao-quan LIU.

Phn 86-25-8327-1260.

Fax 86-25-8327-1386.

E-mail_Liuxiaoquan_cpu@yahoo.com.cn

Received 2006-03-03

Accepted 2006-04-10

doi: $10.1111 / \mathrm{j} .1745-7254.2006 .00369 . \mathrm{x}$

\begin{abstract}
Aim: To study the metabolism of gambogic acid (GA) and the effects of selective cytochrome P-450 (CYP450) inhibitors on the metabolism of GA in rat liver microsomes in vitro. Methods: Rat liver microsomes were used to perform metabolism studies. Various selective CYP450 inhibitors were used to investigate their effects on the metabolism of GA and the principal CYP450 isoform involved in the formation of major metabolite $\mathrm{M}_{1}$ in rat liver microsomes. Types of inhibition in an enzyme kinetics model were used to model the interaction. Results: GA was rapidly metabolized to two phase I metabolites, $\mathrm{M}_{1}$ and $\mathrm{M}_{2}$, in rat liver microsomes. $\mathrm{M}_{1}$ and $\mathrm{M}_{2}$ were tentatively presumed to be the hydration metabolite and epoxide metabolite of GA, respectively. $\alpha$-Naphthoflavone uncompetitively inhibited the formation of $\mathrm{M}_{1}$ while ketoconazole, sulfaphenazole, diethyl dithiocarbamate and quinidine had little or no inhibitory effects on the formation of $\mathrm{M}_{1}$. Conclusion: GA is rapidly metabolized in rat liver microsomes and $\mathrm{M}_{1}$ is crucial for the elimination of GA. Cytochrome P-450 1A2 is the major rat CYP involved in the metabolism of GA.
\end{abstract}

\section{Introduction}

Gambogic acid (GA) is a natural product isolated from the gamboge resin of Garcinia hanburyi tree in South-east Asia. The resin is used as a traditional medicine and as a color material for painting ${ }^{[1]}$. Gamboge can be used for detoxification, hemostasis, and as a parasiticide ${ }^{[2]}$. GA has been previously reported to have potent cytotoxicities against various cancer cell lines and to be a potent apoptosis inducer.

In one study, anti-tumor activity of general gambogic acids (GGA) in the experimental transplantation tumor SMMC-7721 was evaluated by relative tumor growth ratio. GGA had an inhibitory effect on the growth of SMMC-7721, which might be related to its inhibition of telomerase activity ${ }^{[3]}$. In another study, GA could inhibit the growth of SPC-A1 cells and its tumor xenografts, and when treated with GA for a period of time, telomerase activity and expression of hTERT mRNA in the tumor cells were both inhibited significantly. It is safe, at least in part, to conclude that the downregulating telomerase activity of GA by partly modifying the expression of hTERT mRNA in SPC-A1 cells may be one possible mechanism for the inhibitory activity of GA in the cells ${ }^{[4]}$.
Thus GA is an effective telomerase inhibitor and displays potent anticancer activity both in vitro and in vivo. Moreover, GA was found to induce apoptosis independent of the cell cycle, which is different from paclitaxel that arrests cells in the $\mathrm{G}_{2} / \mathrm{M}$ phase $\mathrm{e}^{[1]}$. The selective induction of apoptosis of GA on MGC- 803 cells were studied and activation of the bax gene and suppression of the bc1-2 gene may contribute to the apoptosis mechanism ${ }^{[5]}$. Another study suggested that GA binding to transferrin receptor induces a unique signal leading to rapid apoptosis of tumor cells ${ }^{[6]}$.

There are several studies about its pharmacodynamic characteristics, while only few comment on the pharmacokinetics. The purpose of these experiments was to investigate the metabolism of GA in rat liver microsomes in vitro and to identify the major CYP450 isoform involved in the metabolism of GA and the inhibition type of selective CYP450 inhibitor on its metabolism.

\section{Materials and methods}

Chemicals Gambogic acid was provided by the Department of Pharmaceutical Chemistry, China Pharmaceutical 
University. Glucose-6-phosphate dehydrogenase (Type V), $\alpha$-naphthoflavone ( $\alpha$-Naph), sulfaphenazole (Sul), quinidine (Qui), and diethyl dithiocarbamate (DDC) were purchased from Sigma Chemical (St Louis, USA). Ketoconazole (Ket) was kindly provided by Nanjing Second Pharmaceutical Factory (Nanjing, China). $\alpha$-Nicotinamide adenine dinucleotide phosphate (NADP) and glucose-6-phosphate(G-6-P) were purchased from Shanghai Lizhudongfeng Biotechnological. (Shanghai, China). All other supplies were of the highest grades available from standard commercial sources.

Tissue samples and preparation of liver microsomes Sprague-Dawley rats (180-220 g; 6-8 weeks of age) were obtained from the experimental animal center of China Pharmaceutical University, and the studies were approved by the Animal Ethics Committee of China Pharmaceutical University. The rat liver microsomes were prepared by differential centrifugation ${ }^{[7]}$. Microsomal protein concentration was determined by the method of Lowry et $a l^{[8]}$.

Incubation and sample preparation The incubation conditions of the experiment were established and controlled to provide a reproducible and linear rate of the metabolite. A typical incubation mixture consisted of potassium phosphate buffer (PH 7.4) $100 \mathrm{mmol} / \mathrm{L}$, an NADPH generating system $\left(\mathrm{MgCl}_{2} 5 \mathrm{mmol} / \mathrm{L}\right.$, G-6-P $10 \mathrm{mmol} / \mathrm{L}$, NADP $1 \mathrm{mmol} / \mathrm{L}$, G-6$\mathrm{PDH} 1 \mathrm{kU} / \mathrm{L}$ ), gambogic acid $40 \mu \mathrm{mol} / \mathrm{L}$, and microsomal protein $1 \mathrm{~g} / \mathrm{L}$, in a final volume of $1 \mathrm{~mL}^{[7]}$. The reaction was initiated by addition of the NADPH generating system. After incubation at $37^{\circ} \mathrm{C}$ for $30 \mathrm{~min}$, the reaction was terminated by adding $200 \mu \mathrm{L}$ of $\mathrm{HCl} 1 \mathrm{~mol} / \mathrm{L}$. GA and its metabolites $\left(\mathrm{M}_{1}\right.$ and $\left.\mathrm{M}_{2}\right)$ in the incubation mixture were determined by HPLC method ${ }^{[9]}$ with felodipine as its internal standard. The reaction mixtures were extracted with $5 \mathrm{~mL}$ of ether and centrifuged at $1000 \times \mathrm{g}$ for $10 \mathrm{~min}$. The organic fraction was evaporated under a gentle stream of air at $45^{\circ} \mathrm{C}$. The residue was dissolved in $100 \mu \mathrm{L}$ of mobile phase and an aliquot $(20$ $\mu \mathrm{L}$ ) was injected onto the Lichrospher $\mathrm{C}_{18}$ column $(250 \mathrm{~mm} \times$ $4.6 \mathrm{~mm}$ ID, $5 \mu \mathrm{m}$, Hanbon, China). These procedures were performed using a Shimadzu HPLC-UV system (Shimadzu Company, Kyoto, Japan) consisting of a LC-10AD $\mathrm{AP}_{\mathrm{VP}}$ pump, a SPD-10AD ${ }_{\mathrm{VP}} \mathrm{UV}$-VIS detector and a CTO-10A column oven. GA and its metabolites in the incubation mixture were then determined by mass analysis ${ }^{[9]}$.

Inhibition study The effects of various selective CYP inhibitors on the formation of metabolite $\mathrm{M}_{1}$ in rat liver microsomes were investigated. The inhibitors studied were $\alpha$ Naph(CYP1A2), Qui(CYP2D1), DDC(CYP2E1), Sul(CYP2C), and Ket (CYP3A). The concentration of GA was $40 \mu \mathrm{mol} / \mathrm{L}$ and the concentration range of inhibitors was $2.5-20 \mu \mathrm{mol} / \mathrm{L}$ for Qui, 12.5-100 $\mu \mathrm{mol} / \mathrm{L}$ for $\alpha-N a p h$ and Sul, 6.25-100 $\mu \mathrm{mol} / \mathrm{L}$ for DDC, and 0.5-5 $\mu \mathrm{mol} / \mathrm{L}$ for Ket.

Study of inhibition type After identifying the major metabolic enzyme of GA in vitro, we incubated GA in rat liver microsomes in the absence and presence of $\alpha$-Naph. Then the types of inhibition in an enzyme kinetics model were used to investigate the inhibition type of $\alpha$-Naph on the formation of $\mathrm{M}_{1}$. The concentration range of GA was 10-100 $\mu \mathrm{mol} / \mathrm{L}$ and $\alpha$-Naph was $12.5-100 \mu \mathrm{mol} / \mathrm{L}$. Multiple linear regression was used for the inhibition study.

\section{Results}

Following incubation of gambogic acid with rat liver microsomes, GA was rapidly metabolized and two metabolites $\left(\mathrm{M}_{1}\right.$ and $\left.\mathrm{M}_{2}\right)$ were isolated in the incubation with the corresponding concentration of GA decreased (Figure 1).
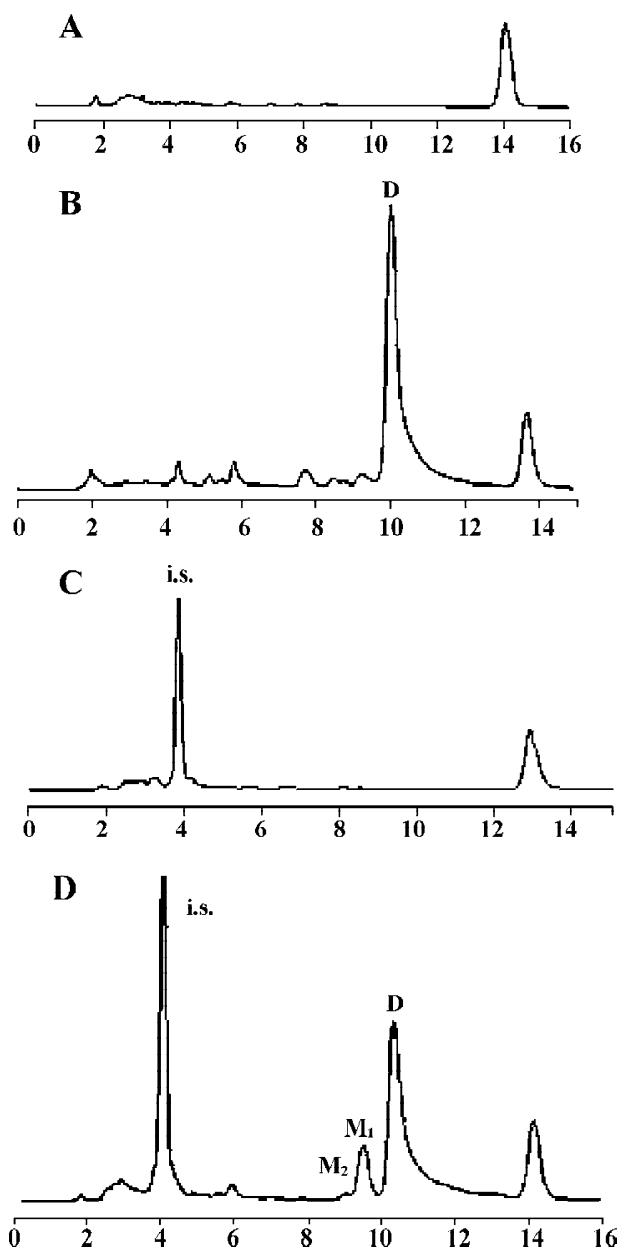

Figure 1. HPLC chromatograms of (A) blank rat liver microsomes, (B) GA standard in blank rat liver microsomes, (C) internal standard in blank rat liver microsomes. (D) Incubation of GA with rat liver microsomes. 
Direct HPLC/MS analysis ${ }^{[9]}$ using an electro-spray ionization interface under positive ion mode resolved two drugrelated compounds in the incubations (Figure 2). $\mathrm{M}_{1}$ exhibited the protonated molecular ion $[\mathrm{M}+\mathrm{Na}]^{+}$at $m / z 669,[\mathrm{M}+\mathrm{H}]^{+}$ at $m / z 647$, and $\mathrm{M}_{2}$ exhibited the protonated molecular ion $[\mathrm{M}+\mathrm{H}]^{+}$at $m / z 645,[\mathrm{M}+\mathrm{Na}]^{+}$at $m / z 667$. Thus, the molecular weight of $M_{1}$ is 646 and $M_{2}$ is 644 . According to HPLC and mass spectra analysis, we can tentatively presume that $M_{1}$ was the hydration metabolite and $\mathrm{M}_{2}$ was the epoxide me- tabolite of GA. But this needs further identification. The proposed metabolic pathway of GA is present in Figure 3.

The effects of inhibitors on the formation of $\mathrm{M}_{1}$ are presented in Figure 4. $\alpha$-Naph, the specific inhibitor of CYP1A2, could inhibit the formation of $\mathrm{M}_{1}$ while other inhibitors had no significant inhibitory effects on the $\mathrm{M}_{1}$ formation (Figure 3 ). The Michaelis-Menten plot of metabolite $M_{1}$ in rat liver microsomes in the absence and presence of $\alpha$-Naph is presented in Figure 5. The inhibition is parallel to the concen-

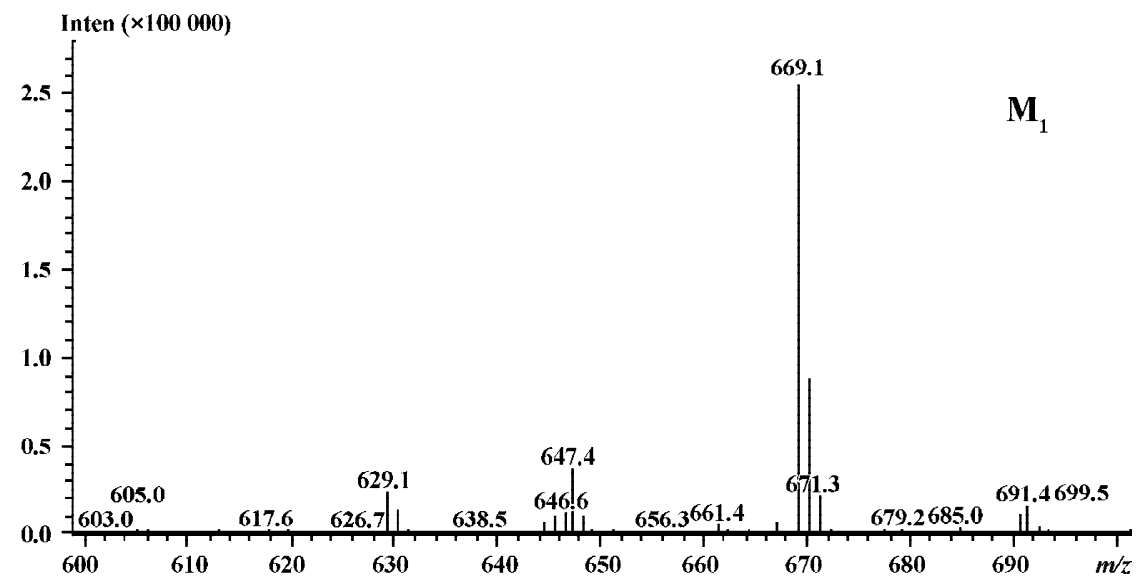

Inten $(\times 100000)$
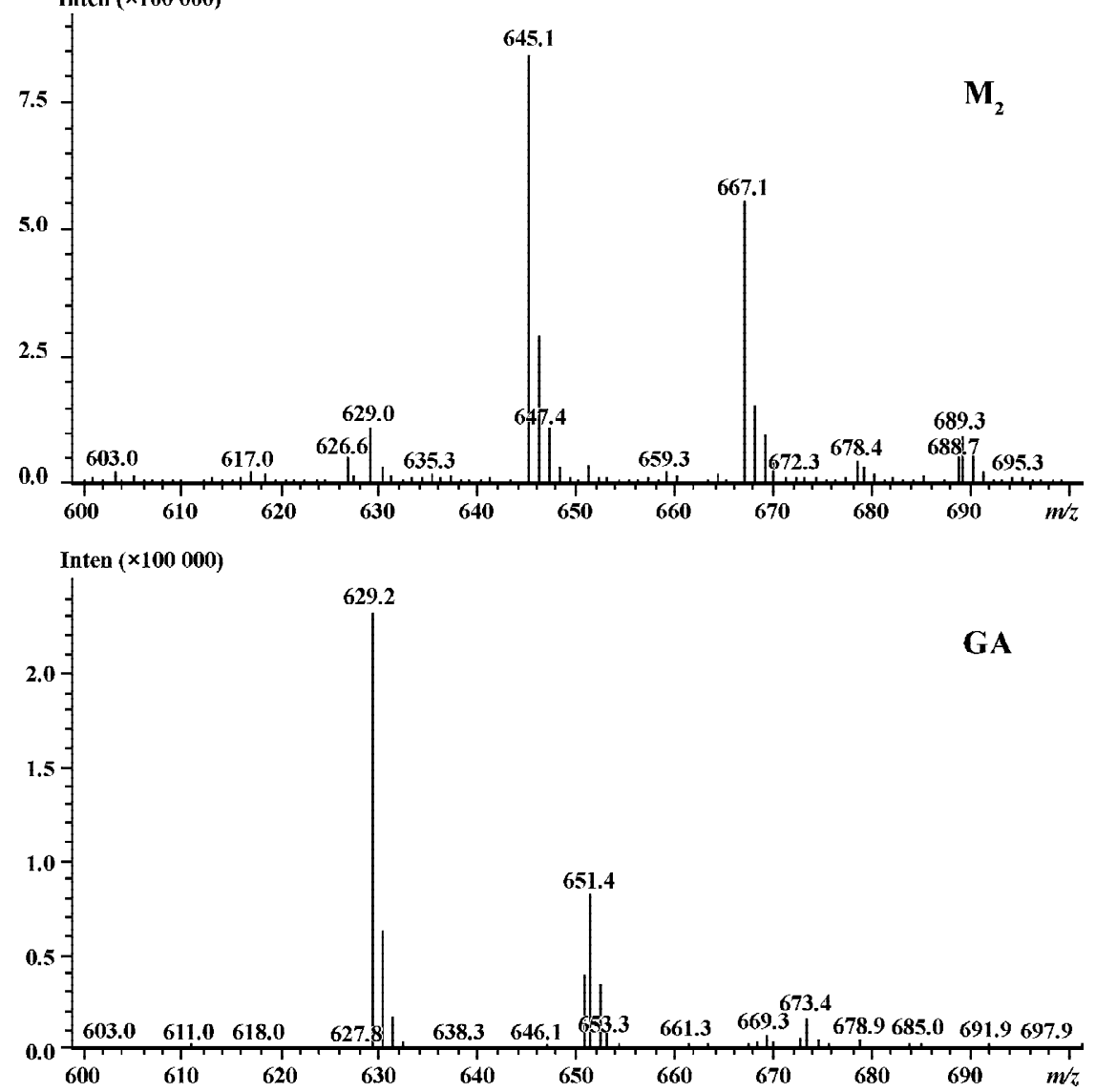

Figure 2. LC/MS spectra of GA and its metabolites in rat liver microsomes. 


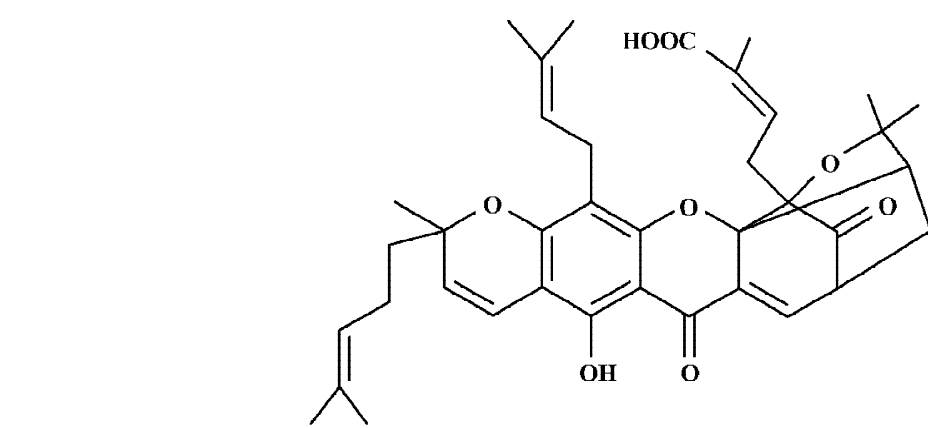

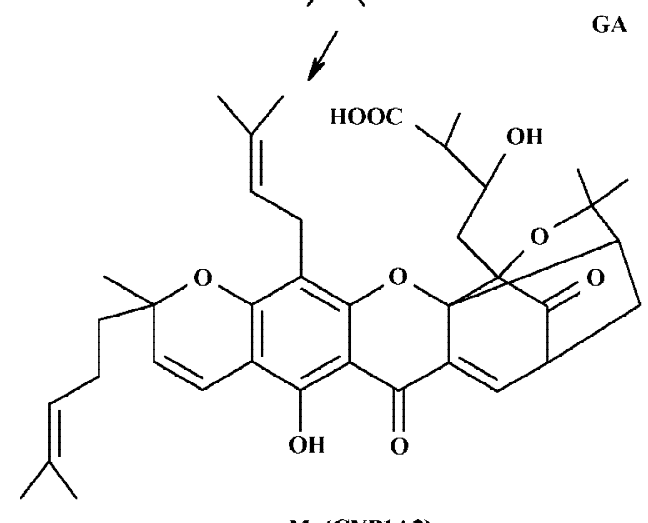

$M_{1}($ CYP1A2)

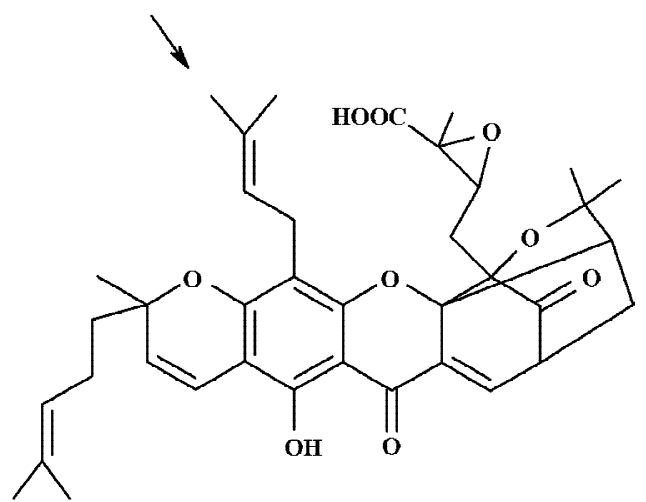

$\mathbf{M}_{2}$
Figure 3. The proposed metabolic pathway of GA in rat liver microsomes.
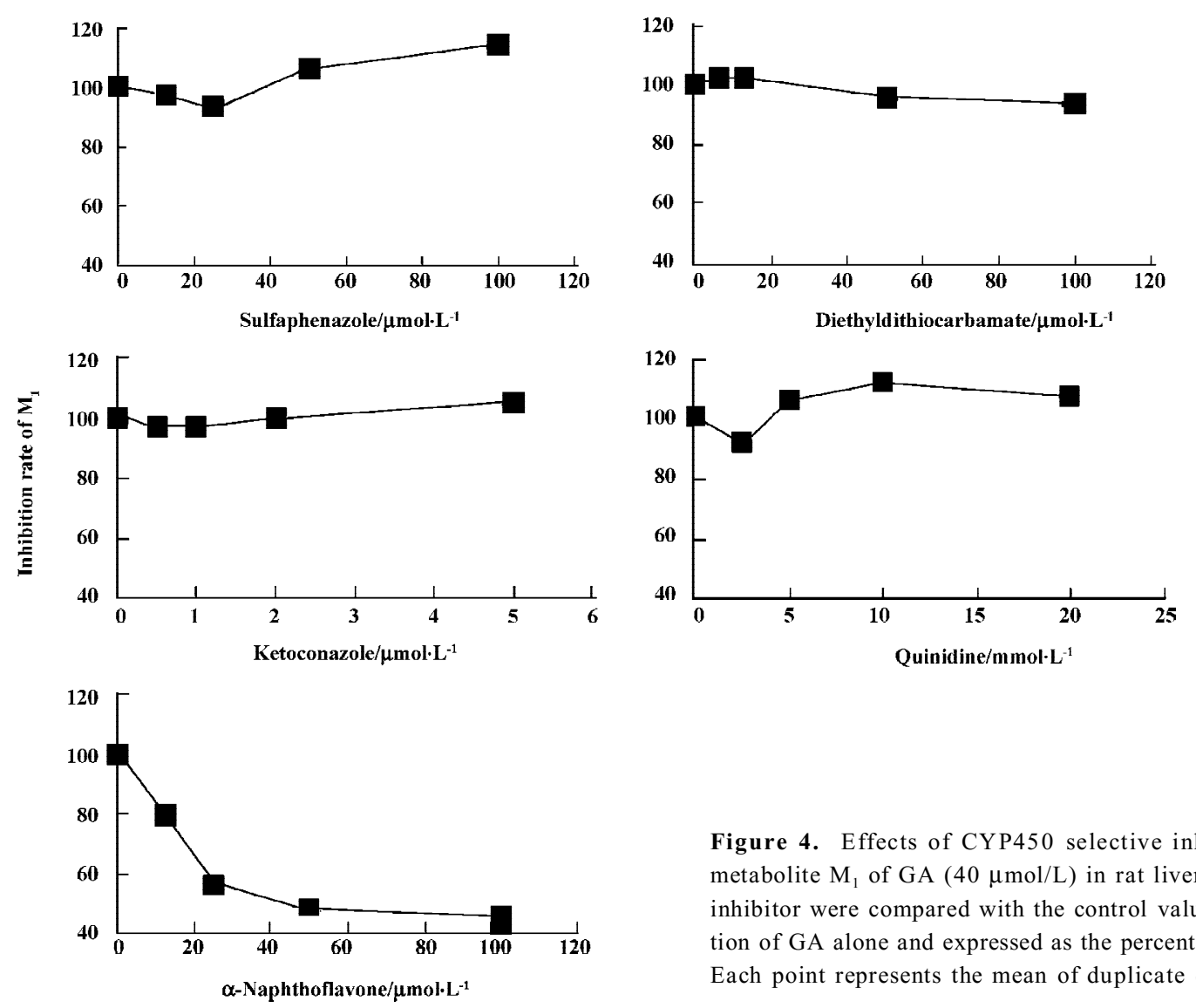

Figure 4. Effects of CYP450 selective inhibitors on the formation of metabolite $\mathrm{M}_{1}$ of GA $(40 \mu \mathrm{mol} / \mathrm{L})$ in rat liver microsomes. Effects of each inhibitor were compared with the control values determined by the incubation of GA alone and expressed as the percentage of control values $(100 \%)$. Each point represents the mean of duplicate determination. 


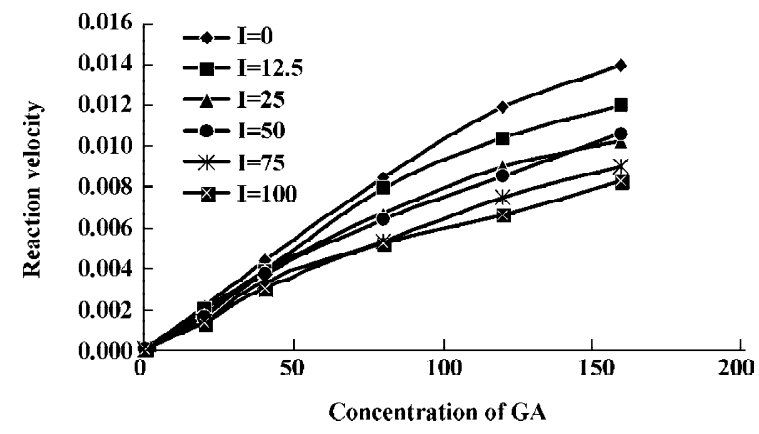

Figure 5. The Michaelis-Menten plot of GA in rat liver microsomes in the absence and presence of $\alpha$-naphthoflavone.

trations of both inhibitor and substrate, suggesting that the inhibition conforms to noncompetitive or uncompetitive types (Figure 5). Two types of inhibition in an enzyme kinetics model were then used to model the interaction: (1) the noncompetitive type, and (2) the uncompetitive type (Figure 6).

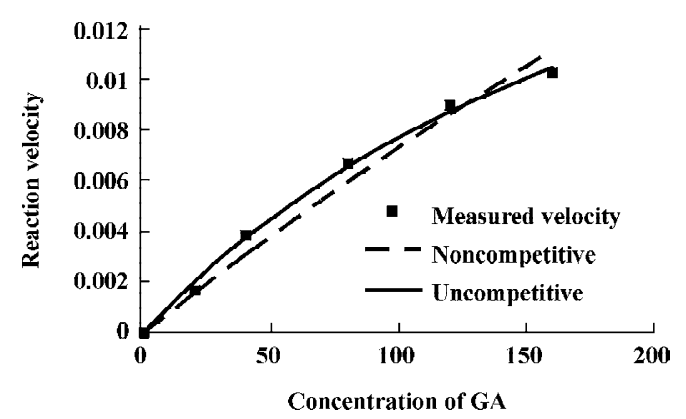

Figure 6. Two types of inhibition in an enzyme kinetics model were used to model the interaction.

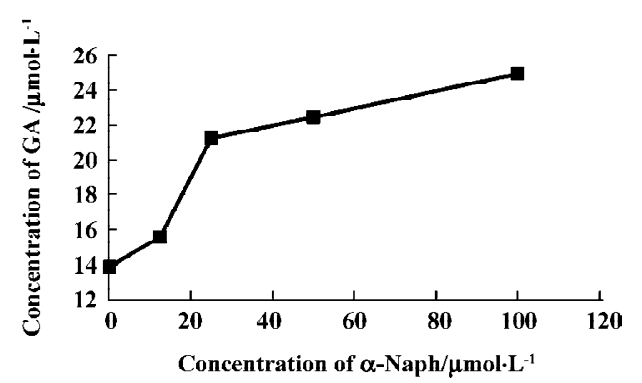

Figure 7. Effect of $\alpha$-naphthoflavone on the metabolism of GA (40 $\mu \mathrm{mol} / \mathrm{L})$ in rat liver microsomes. Each point represents the mean of duplicate determination.

As shown in Figure 6, $\alpha$-Naph uncompetitively inhibited the formation of $\mathrm{M}_{1}$, resulting in a lowered rate of GA metabolism in rat liver microsomes (Figure 7).

\section{Discussion}

The results of present studies indicated that GA was rapidly metabolized in rat liver microsomes. Two metabolites were isolated and identified in the incubation mixture. In the control experiments without NADPH, only microsome and GA, there are no metabolites formed in the incubation. This suggests that the formation of $\mathrm{M}_{1}$ is NADPH-dependent. $\mathrm{M}_{1}$ was one of the major metabolites in the incubation and it was proposed to be the hydration metabolite of GA. Inhibition of the formation of this metabolite would directly result in lowing of the metabolic rate of GA. This suggested that the formation of $M_{1}$ is crucial for the elimination of GA. $M_{2}$ was proposed to be the epoxide metabolite of GA. Our previous studies ${ }^{[8]}$ have shown that GA could be metabolized to four metabolites in rat bile in vivo, including two phase I metabolites $\left(\mathrm{M}_{1}\right.$ and $\left.\mathrm{M}_{2}\right)$ and two phase II metabolites $\left(\mathrm{M}_{3}\right.$ and $\mathrm{M}_{4}$ ), which were presumed to be the phase II metabolites of $\mathrm{M}_{1}$ and $\mathrm{M}_{2}$, respectively. The HPLC and LC-ESI-MS chromatographic behaviors of $\mathrm{M}_{1}$ and $\mathrm{M}_{2}$ in vitro studies showed good correlations with in vivo results.

$\alpha$-Naph could uncompetitively inhibit the formation of $\mathrm{M}_{1}$ and lower the metabolism of GA, while other inhibitors for CYP3A (Ket), CYP2C (Sul), CYP2D1 (Qui) and CYP2E1 (DDC) had little or no effect on the formation of $\mathrm{M}_{1}$. The results suggest that CYP1A2 is a major CYP450 isoform involved in the metabolism of GA. Pharmacokinetic drug interactions caused by metabolic processes are regarded as one of the most important factors affecting the concentration of drugs ${ }^{[10]}$. Therefore, inhibition of the above metabolic pathway can lower the formation rate of $M_{1}$ and increase the concentration of GA, suggesting the possibilities of metabolic interactions of GA with other therapeutic agents, such as inhibitors and substrates of CYP1A2. In clinical applications, drug-drug interactions often occur when two or more drugs are used in combination, which can result in severe side effects in some cases ${ }^{[11]}$. This is worth noting when GA is used in combination with other therapeutic agents that may have metabolic interactions with it. All of the above results contribute to our understanding of the metabolism of GA in rats.

\section{References}

1 Zhang HZ, Kasibhatla S, Wang Y, Herich J, Guastella J, Tseng B, et al. Discovery, characterization and SAR of gambogic acid as a potent apoptosis inducer by a HTS assay . Bioorg Med Chem 2004; 12: 309-17.

2 Liu W, Guo QL, Li Z, Gu HY. Anticancer effect and apoptosis induction of gambogic acid in human gastric cancer line BGC823. World J Gastroenterol 2005; 11: 3655-9. 
3 Guo QL, You QD, Wu ZQ, Yuan ST, Zhao L. General gambogic acids inhibited growth of human hepatoma SMMC-7721 cells in vitro and in nude mice. Acta Pharmacol Sin 2004; 25: 769-74.

4 Wu ZQ, Guo QL, You QD, Zhao L, Gu HY. Gambogic acid inhibits proliferation of human lung carcinoma SPC-A1 cells in vivo and in vitro and represses telomerase activity and telomerase reverse transcriptase mRNA expression in the cells. Biol Pharm Bull 2004; 27: 1769-74.

5 Zhao L, Guo QL, You QD, Wu ZQ, Gu HY. Gambogic acid induces apoptosis and regulates expression of Bax and Bcl-2 protein in human gastric carcinoma MGC-803 cells. Biol Pharm Bull 2004; 27: 998-1003.

6 Kasibnatla S, Jessen KA, Maliartchouk S, Wang JY, English NM, Drewe $\mathrm{J}$, et al. A role for transferrin receptor in triggering apoptosis when targeted with gambogic acid. Cell Biol 2005; 102: 12095-100
7 Liu XQ, Zhao Y, Li D, Qian ZY, Wang GJ. Metabolism and metabolic inhibition of clinipine in human liver microsomes. Acta Pharmacol Sin 2003; 24: 263-8.

8 Dawson JM, Heatlie PL. Lowry method of protein quantification: evidence for photosensitivity. Anal Biochem 1984; 140: 391-3.

9 Hao K, Liu XQ, Wang GJ. Pharmacokinetics of gambogic Acid in rats. J China Pharm Univ 2005; 36: 338-41. Chinese

10 Nakajima M, Inoue T, Shimada N, Tokudome S, Yamamoto T, Kuroiwa Y. Cytochrome P4502C9 catalyzes indomethacin $O$ demethylation in human liver microsomes. Drug Metab Dispos 1998; 26: 261-6.

11 Bjornsson T, Callaghan J, Einolf H, Fischer V, Gan L, Grimm S, et al. The conduct of in vitro and in vivo drug-drug interaction studies: A Pharmaceutical Research and Manufacturers of America (PhRMA) perspective. Drug Metab Dispos 2003; 31: 815-32. 